\title{
Distributed Antenna Systems in Fractional-Frequency-Reuse-Aided Cellular Networks
}

\author{
Jie Zhang, Rong Zhang, Member, IEEE, Guangjun Li, Member, IEEE, and Lajos Hanzo, Fellow, IEEE
}

\begin{abstract}
Distributed antenna system (DAS)-aided unity frequency reuse (UFR) and fractional frequency reuse (FFR) transmission scenarios are investigated in this paper, employing the classic multiobjective of nondominated sorting genetic algorithm II (NSGA-II) for maximizing cell throughput and the coverage. More specifically, coordinated multipoint (CoMP) cooperation is invoked among the distributed antennas (DAs) and the base station (BS) in support of the mobile stations (MSs) roaming at the cell edge, while considering a range of practical impairments. We demonstrate that the received signal-to-interference ratio (SIR) of non-CoMP transmissions follows the lognormal distribution by taking into account both fast fading and large-scale shadowing and path-loss effects. Our simulation results demonstrate that DAS-aided cooperation is capable of achieving a fivefold increased throughput over that of the traditional arrangement. Explicitly, an average throughput per channel of $6.61 \mathrm{bits} / \mathrm{symbol}$ may be achieved.
\end{abstract}

Index Terms-Cellular network, distributed antenna systems (DAS), fractional frequency reuse (FFR).

\section{INTRODUCTION}

$\mathbf{T}$ HE performance of cellular systems is typically constrained by cochannel interference (CCI) [1]-[3]. This is particularly damaging in the cell-edge area of systems employing the radical unity frequency reuse (UFR) philosophy, i.e., when the same frequency is reused within each cell. A straightforward practical solution for avoiding the strong CCI imposed by the adjacent cells is to increase the frequency reuse factor (FRF) by only reusing the same frequency band in cells

Manuscript received June 7, 2012; revised August 28, 2012 and October 11, 2012; accepted November 16, 2012. Date of publication November 29, 2012; date of current version March 13, 2013. This work was supported in part by the Chinese Scholarship Council, by the Research Councils UK under the auspices of the India-UK Advanced Technology Centre, and by the Engineering and Physical Sciences Research Council under the China-UK Science Bridge. L. Hanzo would like to thank the European Research Council under the Advanced Fellow Grant for their financial support. The review of this paper was coordinated by Prof. M. A. Hamouda.

J. Zhang is with the School of Communication and Information Engineering, University of Electronic Science and Technology of China, Chengdu 611731, China, and also with the School of Electronics and Computer Science, University of Southampton, Southampton SO17 1BJ, U.K. (e-mail: jz4@ecs. soton.ac.uk).

R. Zhang and L. Hanzo are with the School of Electronics and Computer Science, University of Southampton, Southampton SO17 1BJ, U.K. (e-mail: rz@ecs.soton.ac.uk; 1h@ecs.soton.ac.uk).

$\mathrm{G}$. Li is with the School of Communication and Information Engineering, University of Electronic Science and Technology of China, Chengdu 611731, China (e-mail: gjli@uestc.edu.cn).

Color versions of one or more of the figures in this paper are available online at http://ieeexplore.ieee.org.

Digital Object Identifier 10.1109/TVT.2012.2230282 that are sufficiently far apart. The FRF $\kappa$ characterizes the frequency reuse (FR) pattern, implying that each cell has access to the fraction $1 / \kappa$ of the entire frequency band. Naturally, this arrangement improves the signal-to-interference-noise ratio (SINR), particularly in the cell-edge area, albeit at the cost of a low area spectral efficiency (ASE).

A smarter way of using the available frequency band is constituted by the hard fractional frequency reuse (hard-FFR, or strict-FFR) technique ${ }^{1}$ [5], which improves the ASE of classic FR, while maintaining a high SINR in the cell-edge area. The philosophy of hard-FFR is that each cell is divided into a cell-center area having access to the cell-center's frequency band $F_{c}$ and the cell-edge area having access to a third of the remaining frequency band $F_{e}$. Thus, hard-FFR corresponds to a combination of UFR and FR in conjunction with an FRF of $\kappa=3$ at the cell edge.

The employment of distributed antenna systems (DAS) in the cell-edge area is capable of further improving the ASE. This is because the DAS may provide a shorter link between the distributed antenna elements (DAEs) and the mobile station (MS) than that provided by the base station (BS). Second, the DAS is capable of supporting more than one MS within a given frequency band at any instant by exploiting their angular separation [6], [7]. We thus refer to the former benefit as the reduced path-loss gain and to the latter as multiplexing gain. However, when achieving a multiplexing gain is sought, the MSs roaming near the angle halfway between two DAEs still suffer from strong multiuser interference (MUI) [8]. In this scenario, the multiple-input-multiple-output coordinated multipoint (CoMP) transmission [9]-[12] constitutes a promising technique of mitigating MUI. However, downlink (DL) CoMP transmission [13], [14] requires full channel state information (CSI) for all the links for the sake of approaching the theoretical upper-bound performance. This can only be achieved by imposing a high pilot overhead for the sake of accurate DL channel estimation at the MS, when frequency-division duplexing is assumed, where the uplink and DL CSI tends to be different. Naturally, the presence of imperfect CSI at the transmitters will erode the efficiency of this MUI mitigation technique [15], [16].

\footnotetext{
${ }^{1}$ This terminology is used in this paper to distinguish it from the soft-FFR [4] design, where one third of the total available frequency is used in the cell-edge area, which is orthogonal to the cell-edge area of the adjacent tier-one cells. On the other hand, the remaining two thirds of the frequency band is reserved for the cell-center area, while relying on a reduced transmission power to avoid strong CCI.
} 


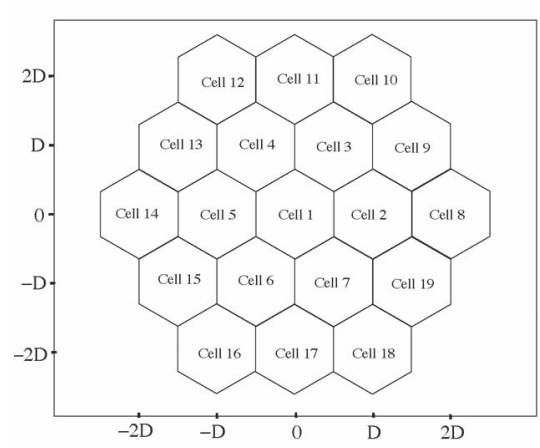

Fig. 1. Cellular system and DAS topology considered.

Against this background, our contributions in this paper are as follows.

- We comprehensively investigate a range of practical DAS deployments in optimally designed FFR-aided cellular networks, both with and without CoMP transmissions. For completeness, we also investigate DASs in traditional cellular networks using similar CoMP transmission techniques as a function of the FRF $\kappa$.

- The powerful multiobjective nondominated sorting genetic algorithm II (NSGA-II) [17] is invoked both for maximizing the achievable cell throughput and for maximizing the cellular coverage simultaneously.

We organize our paper as follows: In Section II, our system model and assumptions and the channel model are described. Then, the idealized UFR transmission, the conventional $\mathrm{FR}^{2}$ transmission, and the realistic FFR compromise scheme are discussed in Section III. The attainable system performance of different scenarios is investigated in Section IV. Finally, we conclude in Section V.

\section{System Description}

Before considering the full list of transmission scenarios, let us highlight our cellular architecture and our composite fading channel model.

\section{A. Cellular System Topology}

We consider the cellular network in Fig. 1, where 19 hexagonal cells are employed, and $N_{a}=6$ DAEs are used within the cell-edge area of each cell. Observe from the left subfigure in Fig. 1 that variable $D$ denotes the intercell distance. In an FR-DAS scenario, the total available bandwidth $F$ is equally divided into three orthogonal frequency bands according to $F=F_{1}+F_{2}+F_{3}$, where each of the frequency bands $F_{i}, i \in$ $1,2,3$ is used in the three adjacent macrocells, respectively. When considering the FFR-DAS scenario, the total available

\footnotetext{
${ }^{2}$ The terminology of FR always has to be associated with the specific FRF $\kappa$, but in the interest of brevity in the rest of this treatise, we typically specify $\kappa$ only, if it is different from $\kappa=3$.
}

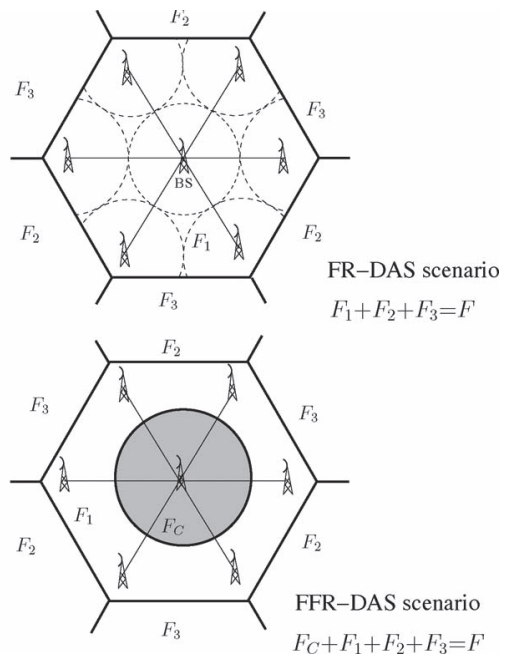

bandwidth $F$ is partitioned into four orthogonal frequency bands, namely, $F_{c}, F_{1}, F_{2}$, and $F_{3}$, obeying $F=F_{c}+F_{1}+$ $F_{2}+F_{3}$, where $F_{c}$ denotes the frequency band available for the cell-center of each cell, and $F_{i}, i \in 1,2,3$ is reserved for the cell-edge area. Furthermore, $\mathbb{B}_{T 1}$ and $\mathbb{B}_{T 2}$ denote the sets containing the six adjacent tier-one cells and 12 tier-two cells, respectively. We also assume that each cell is equally divided into $N$ grid sectors, each supporting a single-antenna-aided MS. Similarly, the assumption of a single transmit antenna is stipulated for the BS and DAEs. The power consumption $P_{t}$ per user is assumed to be equal for the sake of fair comparison.

Since the DAEs are linked to the BS by optical fiber, the assumption of near-perfect reception of the BSs' data at the DAEs becomes realistic. Naturally, having a digital radio-overfiber (DRoF) BS-DAE link is more reliable than a microwave backhaul, albeit it is also more costly. Furthermore, DRoF techniques are capable of avoiding both the intermodulation distortions of the optical fiber and the nonlinearity imposed by the optical components, albeit again, this is typically achieved at increased complexity and cost. Although initially we will exploit the idealized simplifying assumption of a perfect BS-DAE fiber link, we will eliminate this in our future work.

\section{B. Channel Model}

The wireless channel considered is constituted by the three components encapsulated in $h=\sqrt{\Omega \psi} h^{f}$. The Rayleigh fast fading envelope $h^{f}$ is modeled by a zero-mean unity-variance complex Gaussian process, whereas the squared envelope $\left|h^{f}\right|^{2}$ obeys the chi-squared distribution having a degree of freedom of 2 . The path-loss model is given by $\Omega=\Omega_{\beta} d^{-\Omega_{\alpha}}$, where $\Omega_{\beta}$ and $d$ denote the path loss at the reference distance of $d=$ $1 \mathrm{~m}$ and the distance between the transmitter and the receiver expressed in meters, respectively, whereas $\Omega_{\alpha}$ represents the path-loss exponent. Finally, the large-scale shadowing $\psi$ is typically modeled as a lognormal random variable (RV) having a probability density function (pdf) of

$$
p(\psi)=\frac{\xi}{\sqrt{2 \pi} \sigma_{\psi} \psi} \exp \left[-\frac{\left(10 \log _{10} \psi-\mu_{\psi}\right)^{2}}{2 \sigma_{\psi}^{2}}\right]
$$


where $\xi=10 / \ln 10$, and $\mu_{\psi}$ and $\sigma_{\psi}$ are the mean and the standard deviation of $\psi$, both expressed in decibels. In this paper, the path-loss model of $\left(\Omega_{\beta}, \Omega_{\alpha}\right)=\left(1.35 \times 10^{7}, 3\right)$ and shadowing standard deviation of $\sigma_{\psi}=8 \mathrm{~dB}[18]$ is considered.

Given a transmit power of $P_{t}$, the received power $\gamma$ obeys the composite gamma/lognormal distribution [19], whose pdf is given by

$$
\begin{array}{r}
p\left(\gamma \mid \mu_{\psi}, \sigma_{\psi}\right)=\int_{0}^{\infty} \frac{m^{m} \gamma^{m-1}}{\psi^{m} \Gamma(m)} \exp \left(-\frac{m \gamma}{\psi}\right) \frac{\xi}{\sqrt{2 \pi} \sigma_{\psi} \psi} \\
\times \exp \left[-\frac{\left(10 \log _{10} \psi-\mu_{\psi}\right)^{2}}{2 \sigma_{\psi}^{2}}\right] d \psi
\end{array}
$$

where $m$ denotes the shape parameter of the Gamma distribution, and $\mu_{\psi}=10 \log _{10}\left(P_{t} \cdot \Omega\right)$ represents the average power of the received signal, whereas $\Gamma(\cdot)$ represents the Gamma function. The moment-generating function (MGF) of composite gamma/lognormal distribution may be written as [19]

$$
\begin{aligned}
M_{\gamma}(s)= & \int_{0}^{\infty} \exp (s \psi) \frac{m^{m} \gamma^{m-1}}{\psi^{m} \Gamma(m)} \exp \left(-\frac{m \gamma}{\psi}\right) \frac{\xi}{\sqrt{2 \pi} \sigma_{\psi} \psi} \\
& \times \exp \left[-\frac{\left(10 \log _{10} \psi-\mu_{\psi}\right)^{2}}{2 \sigma_{\psi}^{2}}\right] d \psi \\
\approx & \sum_{i=1}^{N_{g}} \frac{w_{i}}{\sqrt{\pi}} \exp \left[1-10^{\left(\sqrt{2} \sigma_{\psi} z_{i}+\mu_{\psi}\right) / 10} s / m\right]^{-m}
\end{aligned}
$$

where the given approximation is derived from the Gauss-Hermit quadrature [20], whereas $z_{i}$ represents the roots of the $n$th Hermite polynomial $H_{n}(x)$, and the associated weights $w_{i}$ of (3) are provided by the lookup table in [21]. However, the composite RV $\gamma$ of (2) is still impervious to analysis; hence, we introduce further approximation. The composite gamma/lognormal distribution RV of (2) may be approximated by a lognormal RV [22] relying on the parameters of $\mu=\mu_{\psi}+\xi[\psi(m)-\ln (m)] \quad$ and $\sigma^{2}=\sigma_{\psi}^{2}+\xi^{2} \varsigma(2, m)$, where $\psi(\cdot)$ and $\varsigma(\cdot, \cdot)$ denote the Euler psi function and Riemann zeta function [21], respectively. By contrast, the distribution of the sum of RVs obeying the pdf of (2) may be approximated by a lognormal RV. This approximation may be carried out either in two distinct steps or in a single direct step. More specifically, in the two-stage approach, it may be approximated first by a lognormal RV, and then, the sum of lognormal RVs may be approximated by a single lognormal RV using the Fenton-Wilkinson, the Schwartz-Yeh, or the MGF-based methods [22]. Alternatively, in the single-stage approach, the sum of RVs obeying the pdf of (2) may be also directly approximated by a lognormal RV [22], [23], where the MGF of the new lognormal RV is equal to the product of the individual MGFs of the constituent gamma/lognormal RVs.

\section{Distributed Antenna System-Aided DOWNLINK TRANSMISSION}

Before considering our DAS-aided transmission scenarios, both the traditional UFR and other FR transmission scenarios are discussed first, which are treated as our benchmarkers.

\section{A. Benchmarkers}

1) UFR Transmission: In the challenging UFR scenario, each MS is only served by its own anchor BS, whereas the remaining active transmissions are considered as CCI. Hence, the discrete-time model of the received signal and the signal-tointerference ratio (SIR) of MS $j$ may be written as

$$
\begin{aligned}
y_{j}^{u} & =h_{j} x_{j}+\sum_{i \in \mathbb{B}_{T 1} \cup \mathbb{B}_{T 2}} h_{i} x_{i}+n_{j} \\
\gamma_{j}^{u} & =\mathbf{E}\left(\frac{\left|h_{j}\right|^{2}}{\sum_{i \in \mathbb{B}_{T 1} \cup \mathbb{B}_{T 2}}\left|h_{i}\right|^{2}}\right)
\end{aligned}
$$

where $\mathbf{E}(\cdot)$ denotes the expectation operation. The first two terms of the received signal of (4) represent the desired signal and the CCI imposed by the tier-one and tier-two cells. Variable $n_{j}$ denotes the circularly symmetric complex Gaussian noise having a covariance of $N_{0}$. Furthermore, $h_{j}$ describes the single-input-single-output DL channel between the $j$ th BS and the supported MS. Finally, we let $x_{j}$ denote the transmitted signal, which is independent of both the noise and the channel, obeying $\mathbf{E}\left(x_{j} x_{j}^{*}\right)=1$.

2) FR Transmission: In contrast to UFR, in the less challenging FR scenario associated with the FRF of $\kappa=3$, no CCI is imposed by the adjacent tier-one cells. The discrete-time model of the received signal and the corresponding SIR may be written as

$$
\begin{aligned}
y_{j}^{f} & =h_{j} x_{j}+\sum_{i \in \mathbb{B}} h_{i} x_{i}+n_{j} \\
\gamma_{j}^{f} & =\mathbf{E}\left(\frac{\left|h_{j}\right|^{2}}{\sum_{i \in \mathbb{B}}\left|h_{i}\right|^{2}}\right)
\end{aligned}
$$

where $\mathbb{B} \subset \mathbb{B}_{T 2}$ denotes the subset of tier-two cells, which reuse the same frequency band as that of the investigated cell.

3) Hard-FFR Transmission: As for the hard-FFR transmission arrangement, the discrete-time model of the received signal and the SIR of MS $j$ within the cell-center area are the same as that of the UFR transmission, which are given by (4). Similarly, the discrete-time model of the received signal and the SIR of MS $j$ within the cell-edge area are the same as that of the conventional FR scenario, which are given by (5).

\section{B. DAS-Aided Hard-FFR Transmission}

Let us now elaborate further on the family of DAS-aided FFR systems, which constitute a more general scenario than the DAS-aided UFR and DAS-aided FR subclasses to be highlighted in Section III-C.

1) FFR-DAS: The DL within the cell-center area of this DAS-aided hard-FFR (FFR-DAS) scenario is reminiscent of UFR transmissions, where the SIR is given by (4). However, when our DAS is invoked within the cell-edge area of an FFRDAS scenario, $N_{u}^{a}$ MSs may be simultaneously supported by the $N_{a}$ DAEs employing maximum ratio combining transmit preprocessing (MRC-TPP) [24] within the same frequency band. Then, the $N_{a}$ DAEs are equally divided into $N_{u}^{a}$ transmission groups, with each group hosting a single MS. For example, when we have $N_{u}^{a}=N_{a}$, then $N_{u}^{a}$ transmission groups are 
formed, where each group contains a single DAE and supports an MS within the same frequency band at the same time, whereas the transmissions within other groups impose intracell CCI. The discrete-time model of the signal received by MS $j$ roaming in the cell-edge area may be expressed as

$$
y_{j}=\mathbf{h}_{j} \mathbf{I}_{j} x_{j}+\sum_{i=1, i \neq j}^{N_{u}^{a}} \mathbf{h}_{j} \mathbf{I}_{i} x_{i}+\sum_{i \in \mathbb{B}} h_{i} x_{i}+n_{j}
$$

where the channel $\mathbf{h}_{j}=\left[h_{1, j}, h_{2, j}, \ldots, h_{N_{a}, j}\right]$ denotes the joint DL multiple-input-single-output (MISO) channel between all the $N_{a}$ DAEs and the $j$ th MS. The vector $\mathbf{I}_{j} \in$ $\mathbb{C}^{N_{a} \times 1}$ in (6) represents the indicator vector of MS $j$ with the $i$ th element indicating whether DAE $i$ is involved in the transmission to the $j$ th MS. The indicator vector has a unity norm and satisfies $\left\|\mathbf{I}_{j}\right\|_{1}=N_{a} / N_{u}^{a}$, which implies that $N_{a} / N_{u}^{a}$ number of DAEs simultaneously transmit using TPP to the $j$ th MS. Furthermore, the first two terms of (6) represent the received signal and the intracell MUI, whereas the third term denotes the CCI imposed by the tier-two cells using the same frequency band in the cell-edge area. Hence, the average SIR at the input of the $j$ th MS receiver may be written as

$$
\gamma_{j}=\mathbf{E}\left(\frac{\left(\overline{\mathbf{h}}_{j} \circ \mathbf{h}_{j}\right) \mathbf{I}_{j}}{\sum_{i=1, i \neq j}^{N_{u}^{a}}\left(\overline{\mathbf{h}}_{j} \circ \mathbf{h}_{j}\right) \mathbf{I}_{i}+\sum_{i \in \mathbb{B}}\left|h_{i}\right|^{2}}\right)
$$

where (o) denotes the Hadamard product of two vectors, and $(\cdot)$ represents the complex conjugate.

2) CoMP-Aided FFR-DAS: In our CoMP-aided hard-FFRDAS transmission scenario, which is referred to as CoMPFFR-DAS, $N_{a}$ DAEs may cooperatively support the $N_{u}^{a}$ MSs of the cell-edge area, provided that all CSI is available at the transmitters. Hence, the discrete-time model of the signal received by MS $j$ may be written as

$$
y_{j}=\mathbf{h}_{j} \mathbf{t}_{j} x_{j}+\sum_{i=1, i \neq j}^{N_{u}^{a}} \mathbf{h}_{j} \mathbf{t}_{i} x_{i}+\sum_{i \in \mathbb{B}} h_{i} x_{i}+n_{j}
$$

where the first two terms of (8) represent the desired signal of the $j$ th MS and the DL MUI imposed by the simultaneous transmissions to other MSs in the cooperative transmission. The $\mathbf{H}=\left[\mathbf{h}_{1}^{T}, \mathbf{h}_{2}^{T}, \ldots, \mathbf{h}_{N_{u}^{a}}^{T}\right]^{T} \in \mathbb{C}^{N_{u}^{a} \times N_{a}}$ represents the channel matrix between the $N_{a}^{u}$ DAEs and the $N_{u}^{a}$ MSs, where $(\cdot)^{T}$ denotes the transpose operation. Furthermore, $\mathbf{t}_{j} \in$ $\mathbb{C}^{N_{a} \times 1}$ denotes the joint linear TPP vector of the cooperative transmission intended for the $j$ th MS, where the matrix $\mathbf{T}=$ $\left[\mathbf{t}_{1}, \mathbf{t}_{2}, \ldots, \mathbf{t}_{N_{u}^{a}}\right] \in \mathbb{C}^{N_{a} \times N_{u}^{a}}$ represents the TPP matrix of the cooperative transmission.

In this paper, we employ the classic zero-forcing (ZF)-based linear TPP technique, which completely eliminates MUI, provided that perfect CSI is available at the transmitters, albeit this is achieved at the cost of noise amplification. The TPP matrix of $\mathbf{W}=\left[\mathbf{w}_{1}, \mathbf{w}_{2}, \ldots, \mathbf{w}_{N_{u}^{a}}\right]$ has vectorial elements of $\mathbf{w}_{j}, j \in$ $1,2, \ldots, N_{u}^{a}$, which are constituted by the normalized column vectors of $\mathbf{H}^{H}\left(\mathbf{H H}^{H}\right)^{-1}$ [10], where $(\cdot)^{H}$ represents the conjugate transpose operation. Furthermore, the same power is allocated for DL transmission to all MSs, which may be expressed as $\mathbf{Q}=\operatorname{diag}\left(\sqrt{P_{t}}, \sqrt{P_{t}}, \ldots, \sqrt{P_{t}}\right) \in \mathbb{R}^{N_{u}^{a} \times N_{u}^{a}}$. Hence, the TPP matrix $\mathbf{T}$ is formulated as $\mathbf{T}=\mathbf{W Q}$, whereas the SIR of MS $j$ in the cell-edge area may be expressed as

$$
\begin{aligned}
\gamma_{j} & =\mathbf{E}\left(\frac{\left|\mathbf{h}_{j} \mathbf{t}_{j}\right|^{2}}{\sum_{i=1, i \neq j}^{N_{u}^{a}}\left|\mathbf{h}_{j} \mathbf{t}_{i}\right|^{2}+\sum_{i \in \mathbb{B}}\left|h_{i}\right|^{2}}\right) \\
& =\mathbf{E}\left(\frac{\left|\mathbf{h}_{j} \mathbf{t}_{j}\right|^{2}}{\sum_{i \in \mathbb{B}}\left|h_{i}\right|^{2}}\right)
\end{aligned}
$$

where the given equation was derived by exploiting the property of $\mathbf{h}_{j} \mathbf{t}_{i}=0$, according to the ZF TPP criterion.

3) Practical CoMP-FFR-DAS: In the presence of the practical impairments discussed in Appendix A, the ZF TPP technique is no longer capable of perfectly eliminating MUI owing to the imperfect CSI fed back by the MSs; thus, the achievable SIR of idealized CoMP-FFR-DAS transmissions will degrade and, hence, has to be accordingly revised. Specifically, the quantized and, hence, imperfect TPP matrix $\mathbf{T}$ is now uniquely described by the TPP codebook index $\mathbf{c}_{\kappa}$ and by the channel quality information (CQI); hence, $\mathbf{h}_{j} \mathbf{t}_{i}=0$ is no longer satisfied. When considering ZF TPP, the expected SIR of practical CoMP scenarios may then be written as

$$
\gamma_{j}=\mathbf{E}\left(\frac{\left|\mathbf{h}_{j} \mathbf{t}_{j}\right|^{2}}{\sum_{i=1, i \neq j}^{N_{u}^{a}}\left|\mathbf{h}_{j} \mathbf{t}_{i}\right|^{2}+\sum_{i \in \mathbb{B}}\left|h_{i}\right|^{2}}\right) .
$$

\section{DAS-Aided UFR and FR Transmission}

1) UFR-DAS and FR-DAS: Similarly, a DAS may be invoked in the cell-edge area in both UFR and in conventional FR scenarios associated with different FRFs $\kappa$, which are referred to as the UFR-DAS and FR-DAS scenarios, respectively. In this case, a total of $N_{u}^{a}=\left(N_{a}+1\right)$ MSs are supported by the anchor BS and the DAEs within the same frequency band, where each transmission is also affected by all the other active transmissions, which are treated as MUI rather than as useful signals. The discrete-time model of the signal and the SIR of MS $j$ are similar to that of the FFR-DAS scenario, which were given by (6) and (7) in conjunction with $\mathbb{B}=\mathbb{B}_{T 1} \cup \mathbb{B}_{T 2}$ for our UFR-DAS transmission. Furthermore, $\left\|\mathbf{I}_{j}\right\|_{1}=1$ and $\mathbf{g}_{j}=$ $\left[\left|h_{j}\right|^{2},\left|h_{1, j}\right|^{2},\left|h_{2, j}\right|^{2}, \ldots,\left|h_{N_{a}, j}\right|^{2}\right]$ represent the effective channel gain between the anchor BS and our DAEs and MS $j$.

2) CoMP-Aided UFR-DAS and FR-DAS: Again, CoMPaided UFR-DAS and FR-DAS transmissions may be invoked for improving the received SIR of the users roaming at angles between the DAEs and between the anchor BS and the DAEs, which are referred to as CoMP-UFR-DAS and CoMP-FRDAS, respectively. When employing ZF TPP, $N_{u}^{a}=\left(N_{a}+\right.$ 1) MSs are cooperatively served by the BS and by the $N_{a}$ DAEs. The discrete-time model of the received signal and the SIR expressions of MS $j$ are similar to that of the CoMPFFR-DAS scenario, which are given by (8)-(10). Furthermore, $\mathbf{h}_{j}=\left[h_{j}, h_{1, j}, h_{2, j}, \ldots, h_{N_{a}, j}\right] \in \mathbb{C}^{1 \times\left(N_{a}+1\right)}$ represents the joint MISO channel between the BS and the $N_{a}$ DAEs and the $j$ th MS for both the CoMP-UFR-DAS and CoMPFR-DAS scenarios, with $\mathbb{B}=\mathbb{B}_{T 1} \cup \mathbb{B}_{T 2}$ for CoMP-UFR-DAS transmissions. Again, the practical impairments discussed in the Appendix will erode the efficiency of the ZF TPP technique; thus, the perfect cancellation of MUI between the MSs is no 
longer possible; hence, the achievable SIR becomes similar to that of the practical CoMP-FFR-DAS scenario.

\section{Multiobjective Optimization}

Having considered the given comprehensive lists of different DAS-aided options, let us now aim at optimizing the corresponding system arrangements in terms of their throughput and seamless coverage by invoking a genetic algorithm (GA)-aided optimization tool, which will be detailed in Section III-E.

1) Performance Metric: In this paper, we assume that each cell is equally divided into a total of $N=10000$ tiles separated by a rectangular grid, where the MSs are randomly distributed right across the grid. We let $\mathbb{N}$ denote the set of all tiles; hence, the number of elements in $\mathbb{N}$ satisfies $|\mathbb{N}|=N$, where $|\cdot|$ represents the number of elements of a set. Let us first define the achievable average throughput $c_{n}$ of tile $n$ associated with the average received SIR $\gamma_{n}$, which may be written using Shannon's classic formula as

$$
\begin{aligned}
c_{n} & =\mathbf{E}\left[\log _{2}\left(1+\gamma_{n}\right)\right] \\
& \leq \log _{2}\left[1+\mathbf{E}\left(\gamma_{n}\right)\right]
\end{aligned}
$$

where the inequality is derived from Jenssen's Inequality, and the related upper bound is treated as our performance metric.

Only a single MS may be supported by the traditional UFR transmission within a given frequency band, whereas $N_{u}^{a}=$ $\left(N_{a}+1\right)$ MSs are simultaneously supported by the UFR-DAS and CoMP-UFR-DAS within the same frequency band at the same time. Hence, the spatially averaged cell throughput per channel, i.e., $\eta^{u}$, associated with the UFR arrangement may be written as

$$
\eta^{u}=\frac{N_{u}^{a}}{N} \sum_{n \in \mathbb{N}} c_{n}
$$

As for the conventional FR relying on an FRF of $\kappa$, the number of supported active users $N_{u}^{a}$ is the same as that of the UFR scenario, and the corresponding spatially averaged cell throughput per channel $\eta^{f}$ may be written as

$$
\eta^{f}=\frac{N_{u}^{a}}{\kappa} \cdot \frac{1}{N} \sum_{n \in \mathbb{N}} c_{n} .
$$

When hard-FFR transmissions are considered, $N_{u}^{a}$ users are supported in the cell-edge area using the same channel, whereas a single user is served in the cell-center area. We let $\rho=$ $F_{c} / F$ denote the frequency partitioning factor for the hard-FFR scenario associated with $F=F_{c}+F_{e}$ denoting the entire frequency band. Then, the spatially averaged cell throughput per channel $\eta^{h f}$ for hard-FFR transmission may be formulated as

$$
\eta^{h f}\left(r_{\mathrm{th}}, \rho\right)=\frac{\rho}{N_{c}} \sum_{n \in \mathbb{N}_{c}} c_{n}+\frac{(1-\rho) N_{u}^{a}}{\kappa} \cdot \frac{1}{N_{e}} \sum_{n \in \mathbb{N}_{e}} c_{n}
$$

where $\mathbb{N}_{c}$ and $\mathbb{N}_{e}$ represent the subsets of tiles belonging to the cell-center area and to the cell-edge area, respectively. In other words, the set $\mathbb{N}$ is divided into the subsets of $\mathbb{N}_{c}$ and $\mathbb{N}_{e}$, satisfying $\mathbb{N}=\mathbb{N}_{c} \cup \mathbb{N}_{e}$. The number of elements in $\mathbb{N}_{c}$ and $\mathbb{N}_{e}$ satisfies $\left|\mathbb{N}_{c}\right|=N_{c}$ and $\left|\mathbb{N}_{e}\right|=N_{e}$. Furthermore, $r_{\text {th }}$ denotes the radius threshold used for deciding whether tile $n$ belongs to the cell-center area or to the cell-edge area. For example, when the hard-FFR scenario is considered, the MS located within a tile of the cell-center area is served by the BS, whereas the MS located in a tile of the cell-edge area is supported by the DAEs; thus, the average SIR $\gamma$ is a function of radius threshold $r_{\mathrm{th}}$.

Naturally, operators always want to eliminate coverage holes with the aim of providing seamless service. Hence, the coverage holes $\phi$ are used as an additional system performance metric in this paper. A coverage hole occurs for a specific tile when the received SIR $\gamma_{n}$ falls below a predefined threshold of $\gamma_{\mathrm{th}}$. As a result, the normalized coverage holes $\phi$ are defined as the number of tiles in coverage holes divided by the total number of tiles, which is formulated as

$$
\phi=\frac{1}{N} \sum_{n \in \mathbb{N}} I\left[\gamma_{\mathrm{th}}-\gamma_{n}\right]
$$

where

$$
I(x)= \begin{cases}1, & \text { if } x \geq 0 \\ 0, & \text { if } x<0\end{cases}
$$

represents the indicator function, and $N$ is the number of all tiles within the investigated cell.

2) Multiobjective Optimization Problem: A practical system cannot be dynamically configured according to specific user positions. Hence, in this paper, the cells are divided into many tiny tiles with the aim of calculating both the spatially averaged cell throughput and the spatially averaged coverage holes, which are treated as the objective function (OF) of our multiobjective optimization problem. Against this background, the optimum transmission scenario has to maximize the spatially averaged cell throughput while simultaneously minimizing the spatially averaged coverage holes, both by carefully fine tuning frequency partitioning factor $\rho$ and by adjusting radius threshold $r_{\text {th }}$. Once the system configuration is optimized, the MSs will be allocated to specific frequency bands, and their mobility may be managed by the classic handover operation. As a result, the transmission link of users will not be affected by the optimization process. Specifically, the corresponding multiobjective optimization problem may be written as

$$
\begin{array}{ll}
\max & f_{1}\left(r_{\mathrm{th}}, \rho\right)=\frac{\rho}{N_{c}} \sum_{n \in \mathbb{N}_{c}} c_{n}+\frac{(1-\rho) N_{u}^{a}}{\kappa} \cdot \frac{1}{N_{e}} \sum_{n \in \mathbb{N}_{e}} c_{n} \\
\min & f_{2}\left(r_{\mathrm{th}}\right)=\frac{1}{N} \sum_{n \in \mathbb{N}} I\left[\gamma_{\mathrm{th}}-\gamma_{n}\right] \\
\text { s.t. } & \frac{\eta_{e}^{u}}{\eta_{c}^{u}} \leq \frac{\eta_{e}}{\eta_{c}} \leq 1
\end{array}
$$

where $\eta_{c}^{u}=\left(1 / N_{c}\right) \sum_{n \in \mathbb{N}_{c}} c_{n}$ and $\eta_{e}^{u}=\left(1 / N_{e}\right) \sum_{n \in \mathbb{N}_{e}} c_{n}$ denote the average throughput per channel in the cell-center area and the cell-edge area when the radical UFR regime is employed. Similarly, the variables $\eta_{c}=\left(1 / N_{c}\right) \sum_{n \in \mathbb{N}_{c}} c_{n}$ and $\eta_{e}=\left(1 / N_{e}\right) \sum_{n \in \mathbb{N}_{e}} c_{n}$ describe the average throughput per channel in the cell-center area and the cell-edge area when the transmission scenario considered is employed. Finally, the UFR scenario's throughput may be deemed to be the lower bound of the optimization problem, since the DAEs employed in the cell-edge area are expected to improve the average throughput. Furthermore, we artificially introduce the upper limit of unity in (15) by ensuring that the cell-edge area's throughput is no better than that of the cell-center area, which is in line with the traditional user experience. 


\section{E. NSGA-II Algorithm}

In this paper, the classic GA optimization method NSGA-II in [17] is employed for maximizing the cell's throughput $\eta$ while simultaneously minimizing the normalized coverage holes $\phi$. The multiobjective NSGA-II algorithm is applied within the multiobjective optimization problem considered, and the optimization process is provided as follows.

1) Initialization: A population $P_{\mathrm{ini}}$ of solutions is randomly generated, which is composed of $M$ individual solutions $\omega_{m}, m=1, \ldots, M$, where we set $M=20$ in this paper.

2) Evaluation: The average cell throughput $\eta$ and the coverage holes $\phi$ of each individual solution $\omega_{m}$ of population $P_{\text {ini }}$ are evaluated, which are given by (13) and (14), respectively.

3) Iteration: The initial population $P_{\mathrm{ini}}$ is used in the ensuing process as the current population $P_{\text {old }}$, until the number of generations reaches its maximum value of $N_{\max }$. In this paper, we used $N_{\max }=100$.

a) Creation: Generate the new population $P_{\text {new }}$ with the aid of using the standard binary tournament selection, crossover, and mutation operation of the GA [25].

b) Combination: Combine the new population $P_{\text {new }}$ with the current population $P_{\text {old }}$, where the combined set $P=\left\{P_{\text {old }}, P_{\text {new }}\right\}$ is formed as the union of $P_{\text {new }}$ and $P_{\text {old }}$.

c) Nondominated sorting: Evaluate OFs $\eta$ and $\phi$ for each individual of the combined population $P$. For each individual $\omega_{m}$ of the population, the nondomination count is calculated as the number of individuals who dominate the individual $\omega_{m}$. The rank of the individuals with zero nondomination count is set to 1 . Then, we recalculate the nondomination count of the remaining individuals of the population, and the rank of the individuals whose nondomination count is zero is set to 2 . This process continues until all individuals are assigned a rank.

d) Assign crowding distance: To maintain diversity among individuals, the individuals having the same rank in less crowded areas will be selected first. In other words, the individuals having a higher crowding distance of the same rank will be selected with higher priority. Specifically, for each OF of the optimization problem, the OF value of the population is first sorted in descending order. Then, the individuals having the minimum and maximum $\mathrm{OF}$ values are assigned an infinite value as their crowding distance, whereas the other individuals are deemed to have the absolutely normalized distance of two adjacent solutions as their crowding distance. The overall crowding distance is calculated as the sum of the crowding-distance values corresponding to each $\mathrm{OF}$.

e) Extraction: Select the best $M$ individual solutions from the combined population $P$ to generate the current population $P_{\text {old }}$. Specifically, the individual solution $\omega_{m}$ having a lower rank will be selected with higher priority, and the individual solution having a higher crowding distance of the same rank will be selected first.

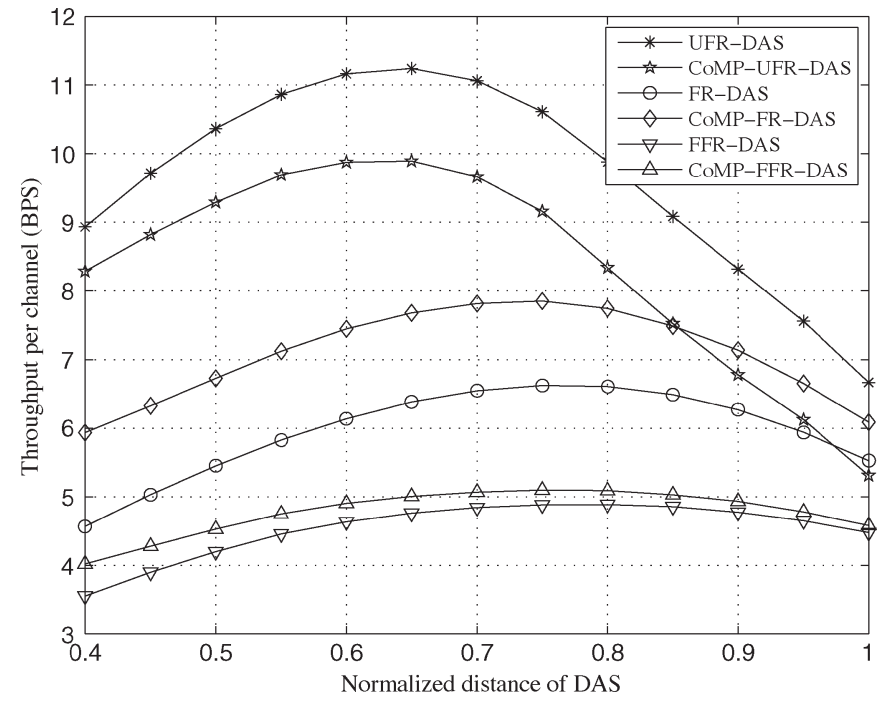

Fig. 2. Attainable throughput per channel versus normalized distance from the BS of DAS for different transmission scenarios when the number of active users supported by the CoMP-FFR-DAS is $N_{u}^{a}=6$.

After the affordable number of iterations, population $P_{\text {old }}$ is treated as the set of optimized solutions for the optimization problem considered.

\section{Performance Evaluation}

Here, we consider a practical wireless cellular system consisting of 19 cells, with a BS-to-BS distance of $D=1000 \mathrm{~m}$ operating in the urban micro setup in [26]. Within each cell, six DAEs are employed in the cell-edge area for the sake of providing a better link between the transmit antenna and the users.

\section{A. Effects of Different DAS Positions}

Fig. 2 characterizes the attainable average throughput ${ }^{3}$ per channel in bits per symbol as a function of different DAS configurations for $N_{u}^{a}=6$, where the DAS configuration was varied by adjusting the radius of the circle on which the DAS elements are positioned. It is clearly shown in Fig. 2 that the throughput of UFR-DAS and CoMP-UFR-DAS transmissions is the highest, when the DAS elements are at $0.65 R$, where $R=D / \sqrt{3}$ denotes the radius of the cell. This is because the cell-edge users suffer from more severe CCI from the DAEs when the DAEs are far from the BS within the adjacent tier-one cells. Furthermore, the achievable throughput per channel of both the FR-DAS and the FFR-DAS scheme reaches its highest value around $r=0.75 R$, which was hence chosen as the default configuration in our forthcoming studies.

\section{B. Effects of CSI Errors}

The TPP matrix employed at the transmitters is a function of the available CSI at the transmitter; thus, the achievable performance of CoMP-aided transmissions depends on both the CSI

\footnotetext{
${ }^{3}$ The achievable throughput defined here and thereafter is the maximum value of the Pareto-optimum front when FFR, FFR-DAS, and CoMP-FFR-DAS transmissions are considered, which are optimized by employing the NSGAII technique. As for the DAS-aided system associated with UFR and FR, the attainable throughput defined here and thereafter mainly depends on the DAS configuration and remains constant for a fixed DAS configuration.
} 

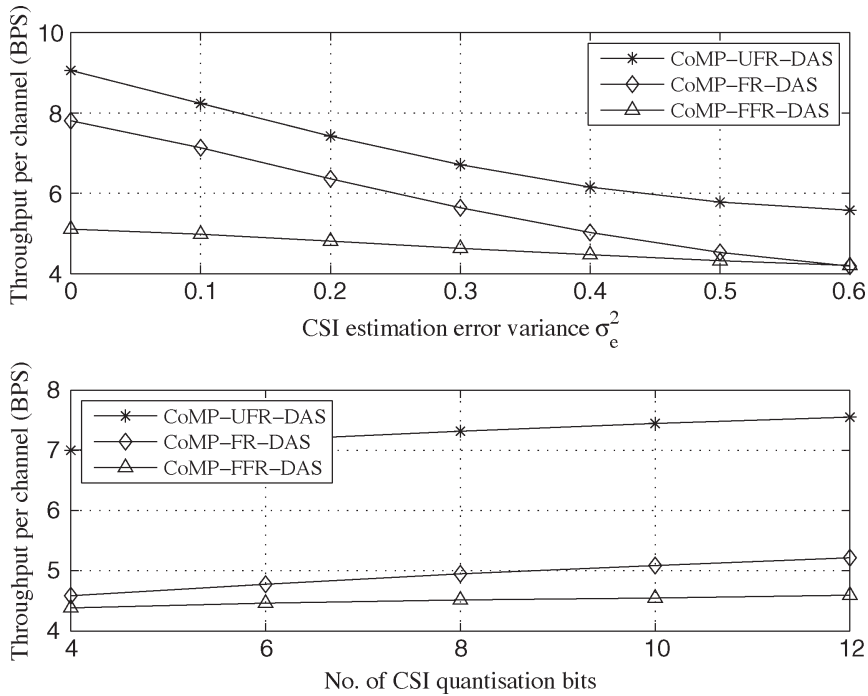

Fig. 3. Attainable throughput per channel versus CSI estimation error variance $\sigma_{e}^{2}$ and versus the number of CSI quantization bits $b$.

estimation accuracy at the receiver and the CSI quantization errors.

1) Effects of CSI Estimation Errors: The upper subfigure in Fig. 3 quantifies the achievable throughput per channel as a function of the CSI estimation error's variance $\sigma_{e}^{2}$ in the absence of CSI quantization errors when the number of MSs supported is $N_{u}^{a}=6$. The attainable throughput of CoMPUFR-DAS, of CoMP-FR-DAS, and of CoMP-FFR-DAS transmissions decays upon increasing the CSI estimation error variance $\sigma_{e}^{2}$. Furthermore, the throughput per channel of CoMP-UFR-DAS and CoMP-FR-DAS transmissions decays faster than that of CoMP-FFR-DAS, because the throughput of CoMP-FFR-DAS partly relies on the UFR transmission within the cell-center area, which is unaware of the CSI errors. Specifically, observe in Fig. 3 that the throughput of CoMPUFR-DAS still remains higher than that of CoMP-FR-DAS and CoMP-FFR-DAS, albeit this is achieved at the cost of reducing the fraction of the cell's area, over which increased throughput is achieved.

2) Effects of Quantization Errors: Let us now investigate the throughput per channel as a function of the number of CSI quantization bits $b$ in the absence of CSI estimation errors, which is shown in the lower subfigure in Fig. 3. Again, as expected, the achievable throughput of CoMP-UFR-DAS, of CoMP-FR-DAS, and of CoMP-FFR-DAS increased when the number of quantization bits $b$ was increased.

\section{Achievable Throughput per Channel and Coverage}

Figs. 4 and 5 show the achievable average throughput per channel and the corresponding fraction of the coverage area (\%) of the different transmission arrangements when the SIR threshold is $\gamma_{\mathrm{th}}=5 \mathrm{~dB}$.

Fig. 4 portrays the achievable throughput of the FFR, FFRDAS, and CoMP-FFR-DAS for both idealized CSI and practical imperfect CSI associated with $\sigma_{e}^{2}=0.05$ and $b=12$ when employing the NSGA-II optimization technique. The Paretooptimum front consisting of $M=20$ individuals is found for both the FFR and the practical CoMP-FFR-DAS scenarios,

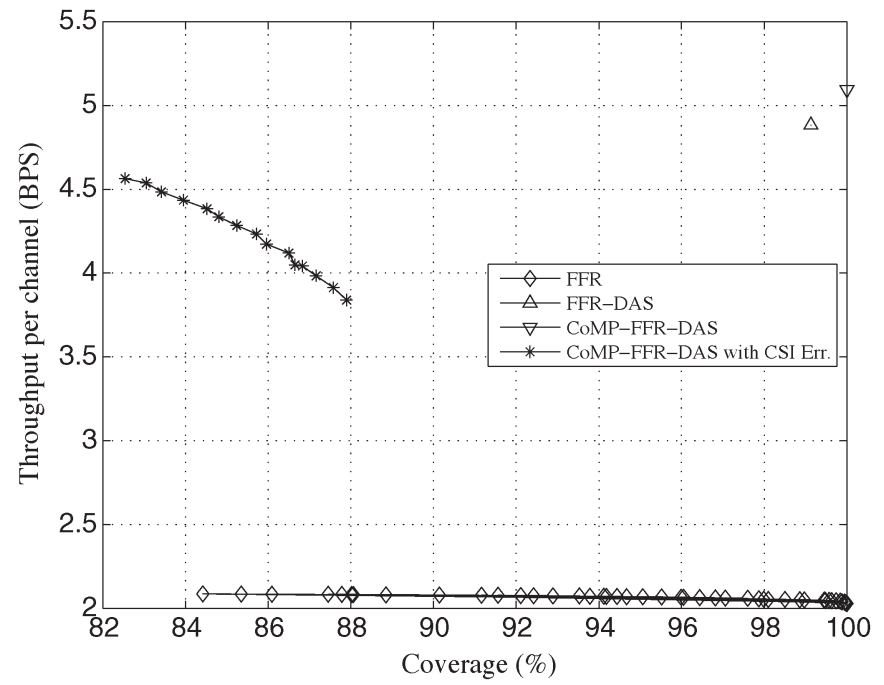

Fig. 4. Achievable Pareto-optimum front for different transmission scenarios for the SIR threshold of $\gamma_{\mathrm{th}}=5 \mathrm{~dB}$ and $N_{u}^{a}=6$.
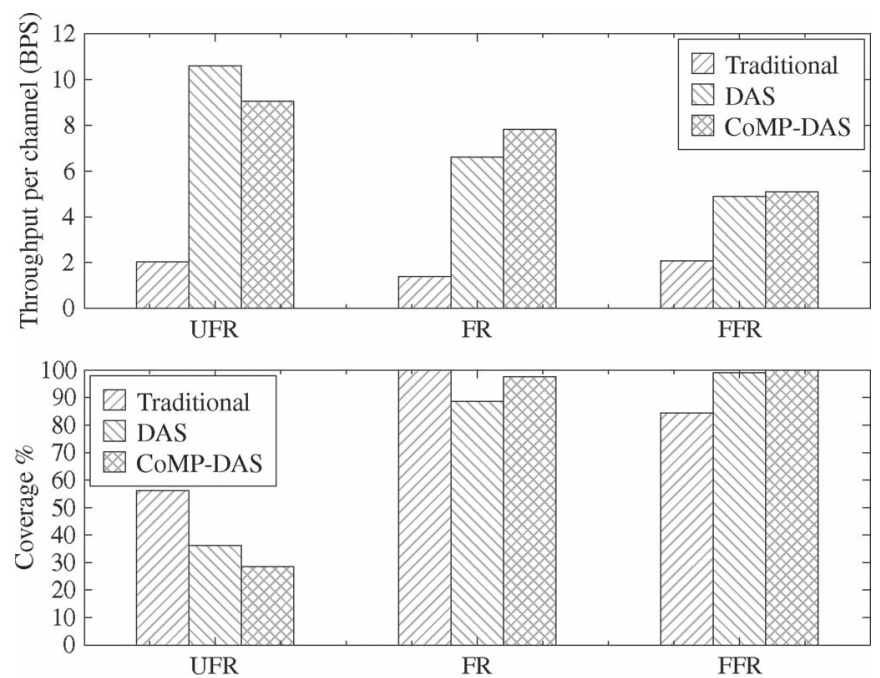

Fig. 5. Achievable throughput per channel (in bits per symbol) and coverage for different transmission arrangements when the SIR threshold is $\gamma_{\mathrm{th}}=5 \mathrm{~dB}$.

which are marked by the star and the diamond in Fig. 4, whereas the Pareto-optimum front of FFR-DAS and of idealized CoMPFFR-DAS without CSI errors contains a single individual candidate solution. More explicitly, the practical CoMP-FFR-DAS transmission scenario is only capable of supporting at most $88 \%$ coverage. This is because the CSI errors encountered erode the efficiency of the CoMP transmission, where $12 \%$ of the tiles of the investigated cell are in outage. Observe in Fig. 4 that the FFR scenario is always capable of supporting at least $84 \%$ coverage, which is a benefit of the cautious FR at the cell edge and path loss in the cell-center area. Furthermore, all individual candidate solutions of both FFR-DAS and the idealized CoMPFFR-DAS transmissions are capable of supporting an almost $100 \%$ coverage, among which the individual having the highest throughput constitutes the Pareto-optimum front, which are marked by the triangles in Fig. 4. As a result, in contrast to the FFR and the CoMP-FFR-DAS with CSI error scenarios, only a single system configuration is found rather than a set of Pareto-optimum system configurations. Observe the plausible fact in Fig. 4 that a higher throughput is achieved at the cost 
of a lower coverage by the FFR and by the practical CoMPFFR-DAS transmission scenarios. It is also clearly shown that both the achievable throughput and the coverage attained by the DAS-aided schemes in the cell-edge area are higher than that recorded in the absence of a DAS.

Fig. 5 shows the achievable spatially averaged throughput per channel and the corresponding coverage for the UFR and FR transmission arrangements when the radius of DAS is $r=0.75 R$. The near-optimum attainable spatially averaged throughput and the corresponding coverage of the FFR, FFRDAS, and CoMP-FFR-DAS schemes is found by using the multiobjective NSGA-II algorithm, where again, the radius of the DAS region is $r=0.75 R$, and the SIR threshold is $\gamma_{\mathrm{th}}=5 \mathrm{~dB}$. Specifically, a throughput per channel of $\eta=2.03$ bits/symbol is achieved by UFR in conjunction with a low coverage of $56 \%$, whereas the traditional FR scheme relying on an FRF of $\kappa=3$ is capable of supporting $100 \%$ coverage at a reduced throughput of $\eta=1.41 \mathrm{bits} / \mathrm{symbol}$. These are treated as our benchmarkers throughout this paper. Although the UFR arrangements are capable of achieving the highest throughput, their relatively poor coverage may preclude their employment. As for the FR-DAS transmission, a high throughput of 6.61 bits/symbol associated with a relatively high coverage of $89 \%$ is achieved in the absence of CoMP transmission. Although a higher throughput and coverage can be attained with the aid of CoMP relying on cooperation between the DAEs and the $\mathrm{BS}$, the achievable throughput was dramatically degraded when considering the practical impairments detailed in the Appendix, which became more serve than that of the FR-DAS scheme.

When hard-FFR transmissions are considered, both high throughput and a high coverage area may be maintained in comparison to both UFR transmission and the FR benchmarker relying on $\kappa=3$. It is important to note that the CoMP-FFRDAS arrangement only has a modest improvement over the FFR-DAS transmission, both of which are capable of supporting an almost $100 \%$ coverage. This is because the CoMP-aided transmission relies on equal-power allocation, albeit a higher throughput would be achievable with the aid of optimal power allocation, user selection, and a more sophisticated TPP technique. However, we set aside these issues for our future work.

\section{Achievable Throughput of All Scenarios Considered}

Table I illustrates the achievable throughput per channel (in bits per symbol) for our different arrangements relying on UFR, FR, and FFR transmissions. Observe from Table I that the throughput of the DAS-aided arrangements may become as high as five times that of the traditional arrangements in both UFR and FR transmissions. A higher throughput may be achieved by the UFR arrangements than that of the FR and FFR schemes, but its relatively low coverage may prevent its employment as a result of the strong CCI emanating from the adjacent tier-one cells. Furthermore, the CoMP-aided arrangements are capable of achieving a higher throughput than that of the schemes dispensing with CoMP transmission. However, the imperfect CSI received at the transmitter side will erode the efficiency of the CoMP transmission, which may hence become even worse than that of the non-CoMP arrangements.
TABLE I

ACHIEVABle ThroughPUT FOR DIFFERENT ARRANGEMENTS IN BITS PER SYMBOL

\begin{tabular}{|l|r|r|r|r|r|}
\hline \multirow{2}{*}{ Arrangements } & \multirow{2}{*}{ UFR } & \multirow{2}{*}{ FR } & \multicolumn{3}{|c|}{ FFR } \\
\cline { 4 - 6 } & & & $N_{u}^{a}=2$ & $N_{u}^{a}=3$ & $N_{u}^{a}=6$ \\
\hline Traditional & 2.03 & 1.41 & 2.09 & 2.09 & 2.09 \\
DAS & 10.59 & 6.61 & 3.33 & 3.93 & 4.88 \\
CoMP-DAS & 9.06 & 7.81 & 3.84 & 4.41 & 5.09 \\
CoMP-DAS with CSI Err. & 7.28 & 5.13 & 3.52 & 4.02 & 4.56 \\
\hline
\end{tabular}

\section{E. Coverage of All Scenarios Considered}

Fig. 6 shows the achievable coverage of UFR-DAS, FR-DAS, and FFR-DAS transmissions for $N_{u}^{a}=6$ when the SIR threshold is $\gamma_{\text {th }}=5 \mathrm{~dB}$. Both figures suggest that, as expected, a high SIR is maintained both around the BS and the $N_{a}$ DAEs, which is an explicit benefit of the low path loss between the transmitter and the receiver. Hence, these transmission scenarios are capable of providing seven hot spots within each cell, where an SIR in excess of $20 \mathrm{~dB}$ is achieved. Since no CCI emanates from the tier-one cells within the FR-DAS and FFR-DAS scenarios, the coverage of both FR-DAS and FFR-DAS is better than that of UFR-DAS. Furthermore, it is also shown in Fig. 6 that the coverage of FFR-DAS is better than that of FR-DAS, which is a benefit of combining the UFR transmissions of the cell-center area with that of FR transmissions in the cell-edge area.

\section{CONCLUSION}

CoMP-aided transmission arrangements employing the classic ZF TPP technique were studied, where the DAEs cooperatively transmit the users' signal. A superior performance was achieved by the DAS-aided transmission arrangements, where the DAS was employed in the cell-edge area to improve the overall system performance. Although CoMP-aided transmissions are capable of achieving a higher throughput and coverage with the aid of idealized perfect CSI at the transmitters' side, the practical CSI impairments dramatically degrade the performance, which, hence, became worse than that of non-CoMP transmissions. Our numerical results demonstrated that the nonCoMP-aided FR-DAS transmission is capable of achieving a throughput per channel of 6.61 bits/symbol in conjunction with a satisfactory coverage of $89 \%$; thus, it is eminently suitable for high-throughput applications. Although the UFR-DAS transmissions are capable of supporting a higher throughput than the FR-DAS scheme, its relatively low coverage area may prevent its employment. However, the performance of CoMPaided transmissions may be improved with the aid of more sophisticated TPP techniques and by more efficient powerallocation schemes.

\section{APPENDIX \\ PRACTICAL IMPAIRMENTS}

The achievable performance of our CoMP transmission scheme is heavily dependent on the accuracy of the precoding matrix, which is a function of the instantaneous CSI. However, in practice, we are unlikely to have the luxury of perfect CSI both at the receiver and at the transmitter due to the combined effects of CSI estimation errors and CSI feedback quantization errors. 


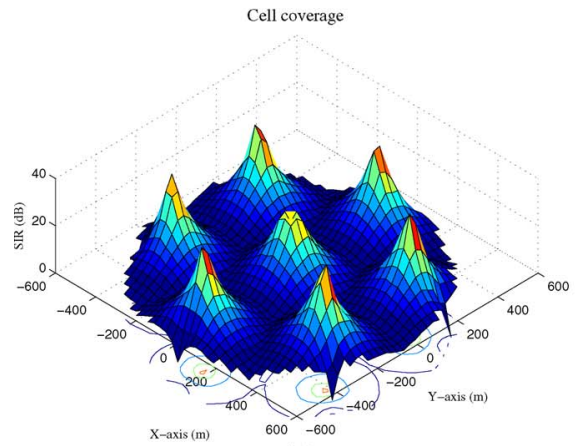

(a)

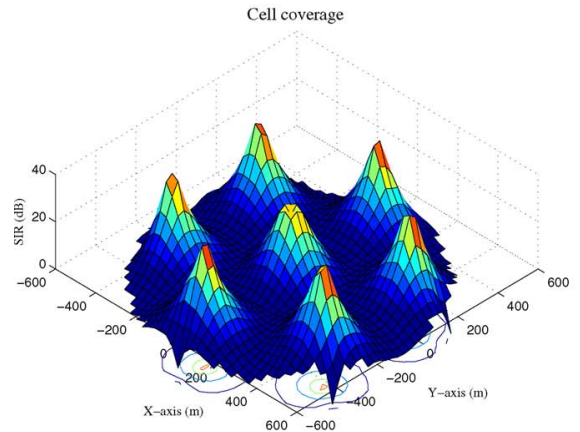

(d)

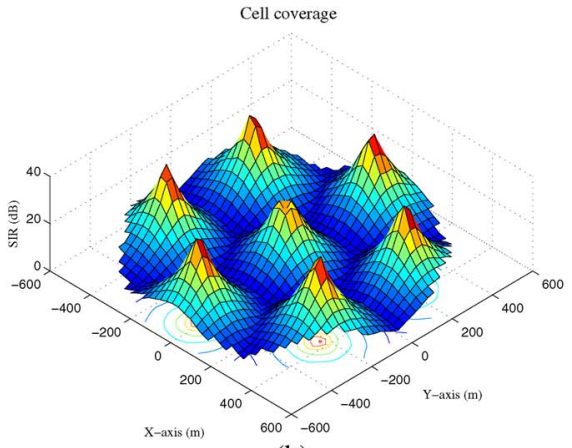

(b)

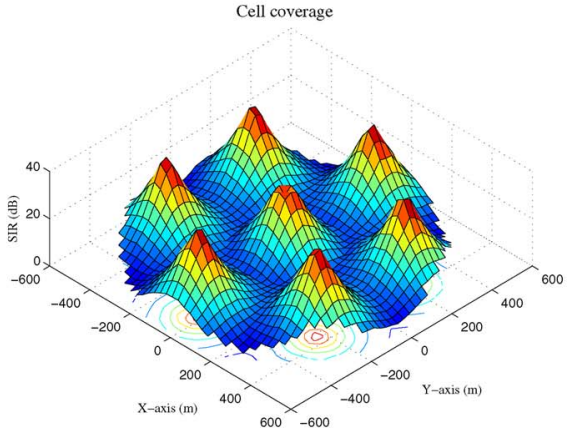

(e)

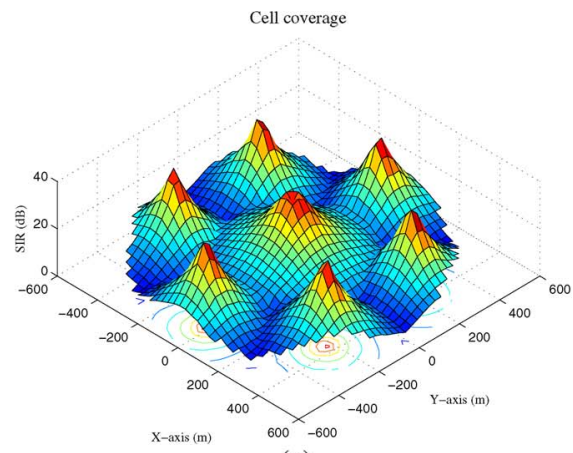

(c)

Cell coverage

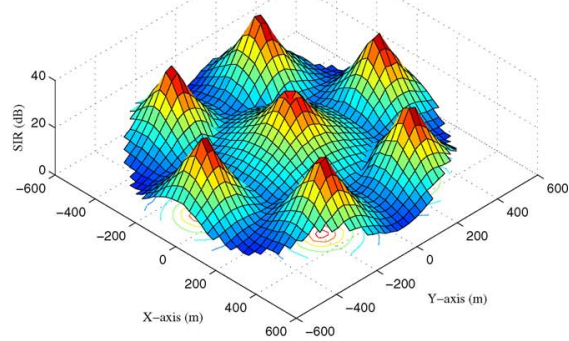

(f)

Fig. 6. Cell coverage for different transmission scenarios associated with DAS for the SIR threshold of $\gamma_{\text {th }}=5$ dB. (a) UFR-DAS. (b) FR-DAS. (c) FFR-DAS. (d) CoMP-UFR-DAS. (e) CoMP-FR-DAS. (f) CoMP-FFR-DAS.

1) CSI Estimation Errors: We assume that MS $j$ is capable of estimating the joint CSI vector $\mathbf{h}_{j}$, subject to the assumption of a Gaussian CSI estimation error having a variance of $\sigma_{e}^{2}$. Then, the channel vector of MS $j$ at the time of estimation may be expressed as $\mathbf{h}_{j}=\hat{\mathbf{h}}_{j}+\sigma_{e} \mathbf{e}_{j}$, where $\mathbf{e}_{j}$ denotes a zeromean unit-variance complex Gaussian vector.

2) CSI Quantization Errors: Having obtained the estimated CSI, we assume the employment of the random vector quantization scheme in [27] and [28] for quantizing the channel direction information (CDI) $\tilde{\mathbf{h}}_{j}=\hat{\mathbf{h}}_{j} /\left\|\hat{\mathbf{h}}_{j}\right\|$, where $\left\|\hat{\mathbf{h}}_{j}\right\|$ represents the CQI, which is assumed to be perfectly fed back to the cooperative DL transmitters. This implies that a CDI quantization codebook $\mathbf{C}=\left\{\mathbf{c}_{1}, \mathbf{c}_{2}, \ldots, \mathbf{c}_{N_{q}}\right\}$ consisting of $N_{q}=2^{b}$ zero-mean unit-norm complex Gaussian vectors is constructed and made available to both the MS and the cooperative transmitters, where $b$ denotes the number of quantization bits, i.e., codebook index bits. In the quantized feedback regime, the $b$ bits representing the particular codebook index of $\kappa_{j}=\max _{q} \cos \theta$, $\theta=\angle\left(\tilde{\mathbf{h}}_{j}, \mathbf{c}_{q}\right)$ are transmitted, where the codebook may be designed to satisfy different design criteria. If we let $\theta_{j}$ denote the angle between the CDI $\tilde{\mathbf{h}}_{j}$ and the quantized vector $\mathbf{c}_{\kappa_{j}}$, then we have $\tilde{\mathbf{h}}_{j}=\mathbf{c}_{\kappa_{j}} \cos \theta_{j}+\mathbf{g}_{\kappa_{j}} \sin \theta_{j}$, where $\mathbf{g}_{\kappa_{j}}$ is a unit vector that lies in the null space of $\mathbf{c}_{\kappa_{j}}$. Furthermore, the expectation of the quantization error $\sin ^{2}\left[\angle\left(\tilde{\mathbf{h}}_{j}, \mathbf{c}_{\kappa_{j}}\right)\right]$ satisfies [29], [30]

$$
\mathbf{E}\left(\sin ^{2} \theta_{j}\right)=2^{b} \cdot \beta\left(2^{b}, \frac{N_{T}}{N_{T}-1}\right) .
$$

Here, $\beta(\cdot, \cdot)$ denotes the beta function, which is defined as $\beta(a$, $b)=\Gamma(a) \Gamma(b) / \Gamma(a+b)$ with $\Gamma(\cdot)$ representing the Gamma function. At the transmitter side, these received codebook-index bits are used for regenerating the quantized CSI by combining them with the perfectly fed back CQI and with codebook $C$. In the presence of both CSI estimation errors and CSI quantization errors, the channel $\mathbf{h}_{j}$ of user $j$ may be written as

$$
\mathbf{h}_{j}=\left\|\mathbf{h}_{j}\right\|\left(\mathbf{c}_{\kappa_{j}} \cos \theta+\mathbf{g}_{\kappa_{j}} \sin \theta\right)+\sigma_{e} \mathbf{e}_{j} .
$$

\section{REFERENCES}

[1] J. G. Andrews, "Interference cancellation for cellular systems: A contemporary overview," IEEE Wireless Commun., vol. 12, no. 2, pp. 19-29, Apr. 2005.

[2] R. Zhang and L. Hanzo, "Wireless cellular networks," IEEE Veh. Technol. Mag., vol. 5, no. 4, pp. 31-39, Dec. 2010.

[3] P. A. Hoeher, S. Badri-Hoeher, W. Xu, and C. Krakowski, "Single-antenna co-channel interference cancellation for TDMA cellular radio systems," IEEE Wireless Commun., vol. 12, no. 2, pp. 30-37, Apr. 2005.

[4] T. D. Novlan, R. K. Ganti, A. Ghosh, and J. G. Andrews, "Analytical evaluation of fractional frequency reuse for OFDMA cellular networks," IEEE Trans. Wireless Commun., vol. 10, no. 12, pp. 4294-4305, Dec. 2011.

[5] T. Novlan, J. G. Andrews, I. Sohn, R. K. Ganti, and A. Ghosh, "Comparison of fractional frequency reuse approaches in the OFDMA cellular downlink," in Proc. IEEE GLOBECOM, Dec. 2010, pp. 1-5.

[6] J. Park, E. Song, and W. Sung, "Capacity analysis for distributed antenna systems using cooperative transmission schemes in fading channels," IEEE Trans. Wireless Commun., vol. 8, no. 2, pp. 586-592, Feb. 2009.

[7] X. You, D. Wang, P. Zhu, and B. Sheng, "Cell edge performance of cellular mobile systems," IEEE J. Sel. Areas Commun., vol. 29, no. 6, pp. 1139-1150, Jun. 2011.

[8] X. Xu, R. Zhang, S. Ghafoor, and L. Hanzo, "Imperfect digital-fiber-opticlink-based cooperative distributed antennas with fractional frequency reuse in multicell multiuser networks," IEEE Trans. Veh. Technol., vol. 60, no. 9, pp. 4439-4449, Nov. 2011.

[9] S. Shamai and B. M. Zaidel, "Enhancing the cellular downlink capacity via co-processing at the transmitting end," in Proc. IEEE VTS 53rd VTC, May 2001, vol. 3, pp. 1745-1749.

[10] H. Zhang and H. Dai, "Cochannel interference mitigation and cooperative processing in downlink multicell multiuser MIMO networks," Proc. EURASIP J. Wireless Commun. Netw., vol. 2004, no. 2, pp. 222-235, Dec. 2004. 
[11] R. Zhang and L. Hanzo, "Cooperative downlink multicell preprocessing relying on reduced-rate back-haul data exchange," IEEE Trans. Veh. Technol., vol. 60, no. 2, pp. 539-545, Feb. 2011.

[12] W. Choi and J. G. Andrews, "Downlink performance and capacity of distributed antenna systems in a multicell environment," IEEE Trans. Wireless Commun., vol. 6, no. 1, pp. 69-73, Jan. 2007.

[13] Q. H. Spencer, A. L. Swindlehurst, and M. Haardt, "Zero-forcing methods for downlink spatial multiplexing in multiuser MIMO channels," IEEE Trans. Signal Process., vol. 52, no. 2, pp. 461-471, Feb. 2004

[14] M. Sadek, A. Tarighat, and A. H. Sayed, "A leakage-based precoding scheme for downlink multi-user MIMO channels," IEEE Trans. Wireless Commun., vol. 6, no. 5, pp. 1711-1721, May 2007.

[15] D. J. Love, R. W. Heath, V. K. N. Lau, D. Gesbert, B. D. Rao, and M. Andrews, "An overview of limited feedback in wireless communication systems," IEEE J. Sel. Areas Commun., vol. 26, no. 8, pp. 1341-1365, Oct. 2008.

[16] M. Huang, S. Zhou, and J. Wang, "Analysis of Tomlinson-Harashima precoding in multiuser MIMO systems with imperfect channel state information," IEEE Trans. Veh. Technol., vol. 57, no. 5, pp. 2856-2867, Sep. 2008.

[17] K. Deb, A. Pratap, S. Agarwal, and T. Meyarivan, "A fast and elitist multiobjective genetic algorithm: NSGA-II," IEEE Trans. Evol. Comput., vol. 6, no. 2, pp. 182-197, Apr. 2002.

[18] T. S. Rappaport, Wireless Communications: Principles and Practice. Englewood Cliffs, NJ: Prentice-Hall, 1996.

[19] M. K. Simon and M.-S. Alouini, Digital Communication over Fading Channels: A Unified Approach to Performance Analysis. New York: Wiley, 2000.

[20] M. Abramowitz and I. A. Stegun, Handbook of Mathematical Functions with Formulas, Graphs, and Mathematical Tables. New York: Dover, 1972.

[21] I. S. Gradshteyn and I. M. Ryzhik, Table of Integrals, Series and Products, 7th ed. New York: Academic, 2007.

[22] J. Zhang and J. G. Andrews, "Distributed antenna systems with randomness," IEEE Trans. Wireless Commun., vol. 7, no. 9, pp. 3636-3646, Sep. 2008

[23] N. B. Mehta, J. Wu, A. F. Molisch, and J. Zhang, "Approximating a sum of random variables with a lognormal," IEEE Trans. Wireless Commun., vol. 6, no. 7, pp. 2690-2699, Jul. 2007.

[24] T. K. Y. Lo, "Maximum ratio transmission," IEEE Trans. Commun., vol. 47, no. 10, pp. 1458-1461, Oct. 1999.

[25] D. E. Goldberg, Genetic Algorithms in Search, Optimization and Machine Learning. Reading, MA: Addison-Wesley, 1989.

[26] R. Steele and L. Hanzo, Mobile Radio Communications. New York: IEEE Press, 1999

[27] W. Santipach and M. L. Honig, "Capacity of a multiple-antenna fading channel with a quantized precoding matrix," IEEE Trans. Inf. Theory, vol. 55, no. 3, pp. 1218-1234, Mar. 2009.

[28] C. K. Au-Yeung and D. J. Love, "On the performance of random vector quantization limited feedback beamforming in a MISO system," IEEE Trans. Wireless Commun., vol. 6, no. 2, pp. 458-462, Feb. 2007.

[29] N. Jindal, "MIMO broadcast channels with finite-rate feedback," IEEE Trans. Inf. Theory, vol. 52, no. 11, pp. 5045-5060, Nov. 2006.

[30] J. Zhang, M. Kountouris, J. G. Andrews, and R. W. Heath, "Multi-mode transmission for the MIMO broadcast channel with imperfect channel state information," IEEE Trans. Commun., vol. 59, no. 3, pp. 803-814, Mar. 2011

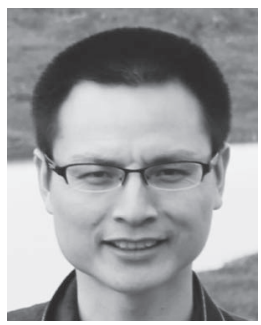

Jie Zhang received the B.Eng. degree in electronics engineering and the M.Eng. degree in signal processing from the University of Electronic Science and Technology of China, Chengdu, China, in 2005 and 2008, respectively, where he is currently working toward the Ph.D. degree with the School of Communication and Information Engineering.

From September 2010 to September 2012, he was a Visiting Student with the Communications Research Group, School of Electronics and Computer Science, University of Southampton, Southampton, U.K. His research interests include cooperative communications and cellular network optimization.

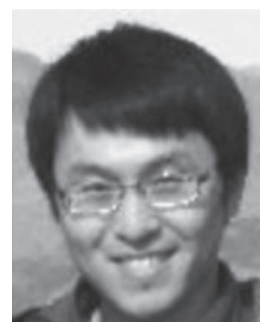

Rong Zhang (M'09) received the B.Eng. degree in communications engineering from Southeast University, Nanjing, China, in 2003 and the M.Sc. (with distinction in radio frequency communications engineering) and the Ph.D. degrees in wireless communications from the University of Southampton, Southampton, U.K., in 2005 and 2009, respectively.

$\mathrm{He}$ was a System Engineer with the Mobile Communications Division of China Telecom. He is currently a Research Fellow with the Communications Research Group, School of Electronics and Computer Science, University of Southampton. He has published 20 journals in the areas of cellular network optimization, multiuser communications, multiantenna communications, and cooperative communications.

Dr. Zhang is a Member of the Institution of Engineering and Technology. He received a joint Engineering and Physical Science Research Council Mobile VCE scholarship in 2006.

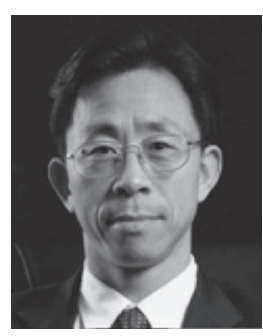

Guangjun Li (M'07) received the M.S. degree from the University of Electronic Science and Technology of China (UESTC), Chengdu, China, in 1985.

Since 1985, he has been with the School of Communication and Information Engineering, UESTC. From 1991 to 1992, he was a Visiting Scholar with RWTH Aachen University, Aachen, Germany. He is currently the Chair of the Communication Integrated Circuits and Systems Engineering Center of UESTC and the Leader of the Wireless Communications and ASIC Laboratory, working on a range of research projects in the field of communication systems sponsored by the National Natural Science Foundation of China, National 863 High Technology Development Project, and National Application-Specific Integrated Circuit (ASIC) Chip Design Project of China. He has published various papers in the area of communication systems, wireless communication networks, embedded systems, ASICs, and silicon-on-chip design.

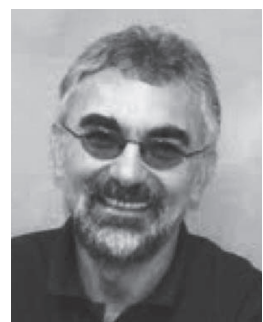

Lajos Hanzo (M'91-SM'92-F'04) received the degree in electronics in 1976 and the Ph.D. degree in 1983. In 2009, he was awarded the honorary doctorate "Doctor Honoris Causa" by the Technical University of Budapest, Budapest, Hungary.

During his 35-year career in telecommunications, he has held various research and academic posts in Hungary, Germany, and the U.K. Since 1986, he has been with the School of Electronics and Computer Science, University of Southampton, Southampton, U.K., where he is currently the Chair in telecommunications. He is a Fellow of the Royal Academy of Engineering, the Institution of Engineering and Technology, and the European Signal Processing Society. He has successfully supervised $80 \mathrm{Ph}$.D. students, coauthored $20 \mathrm{John}$ Wiley/IEEE Press books on mobile radio communications totaling in excess of 10000 pages, published 1300 research entries on IEEE Xplore, acted as both Technical Program Committee and General Chair of IEEE conferences, presented keynote lectures, and has been awarded a number of distinctions Currently, he is directing a 100-strong academic research team, working on a range of research projects in the field of wireless multimedia communications sponsored by industry; the Engineering and Physical Sciences Research Council, U.K.; the European IST Program; and the Mobile Virtual Centre of Excellence, U.K. He is an enthusiastic supporter of industrial and academic liaison and offers a range of industrial courses. His research is funded by the European Research Council's Senior Research Fellow Grant. (For further information on research in progress and associated publications, please refer to http://www-mobile.ecs.soton.ac.uk.)

Dr. Hanzo is a Governor of the IEEE Vehicular Technology Society. During 2008-2012, he was the Editor-in-Chief of the IEEE Press and a Chaired Professor with Tsinghua University, Beijing, China. 